\title{
Homogeneous Time-Invariant Systems
}

\author{
P. P. Vaidyanathan, Fellow, IEEE
}

\begin{abstract}
It is well known that linear time-invariant (LTI) systems produce exponential outputs in response to exponential inputs. The purpose of this letter is to draw attention to the fact that the same result holds for the broader class of homogeneous time-invariant (HTI) systems, though the concept of a frequency response is of little use for such systems.
\end{abstract}

Index Terms-Exponentials, homogeneous time-invariant, linear time-invariant.

\section{INTRODUCTION}

I T IS well known [1], [2] that linear time-invariant (LTI) systems produce exponential outputs in response to exponential inputs. This is usually proved with the help of the convolution sum

$$
y(n)=\sum_{k} h(k) x(n-k) .
$$

Indeed, if $x(n)=a^{n}$ we obtain the output $y(n)=$ $\sum_{k} h(k) a^{n-k}=a^{n} \sum_{k} h(k) a^{-k}=H(a) a^{n}$, where $H(a)$ is the transfer function $H(z)$ evaluated at $z=a$. The purpose of this short letter is to draw attention to a larger class of systems which satisfy this property. While elementary, this result does not appear to be well known.

\section{HTI SYSTEMS}

First recall that linear systems are defined to be those with the following two properties.

1) Homogeneity: If the input $x(n)$ produces output $y(n)$ then $\alpha x(n)$ produces $\alpha y(n)$ for any constant $\alpha$.

2) Superposition Property: If $x_{1}(n)$ and $x_{2}(n)$ produce the outputs $y_{1}(n)$ and $y_{2}(n)$, respectively, then $x_{1}(n)+$ $x_{2}(n)$ produces $y_{1}(n)+y_{2}(n)$.

Systems that satisfy the first property, but not necessarily the second, are called homogeneous systems. If such a system is also time invariant, we call it a homogeneous time-invariant (HTI) system. We now claim that if the input to an HTI system is an exponential $x(n)=a^{n}$, then the output is of the form $H(a) a^{n}$ where $H(a)$ is a constant independent of $n$. [It is not useful to interpret $H($.$) as a transfer function as we shall see$ in Section III.]

Manuscript received September 1, 1998. This work was supported in part by the Office of Naval Research under Grant N00014-93-1-0231. The associate editor coordinating the review of this manuscript and approving it for publication was Dr. R. Shenoy.

The author is with the Department of Electrical Engineering, California Institute of Technology, Pasadena, CA 91125 USA (e-mail: ppvnath@sys.caltech.edu).

Publisher Item Identifier S 1070-9908(99)02457-8.
Proof of the Claim: If an HTI system is not LTI, then we cannot use the convolution sum (1) for the proof. Instead, we go back to the definition. Let $y(n)$ denote the output in response to the exponential input $x(n)=a^{n}$. In view of time invariance, the output in response to the shifted input $x(n+1)=a^{n+1}$ would be $y(n+1)$. But we can also write the shifted input as $x(n+1)=a \cdot a^{n}=a x(n)$. By homogeneity property, we see that the output should also be expressible as $a y(n)$. That is,

$$
y(n+1)=a y(n)
$$

which shows that $y(n)=y(0) a^{n}$. Since $y(0)$ may depend on $a$ as well as the system description, we rewrite this as $y(n)=H(a) a^{n}$.

$\nabla \nabla \nabla$

An Example: Are there HTI systems that are not LTI? Consider the system defined by the following input/output description:

$$
y(n)= \begin{cases}x^{2}(n) / x(n-1), & \text { if } x(n-1) \neq 0 \\ 0, & \text { if } x(n-1)=0\end{cases}
$$

The reader can verify that the superposition property is violated, so this is not a linear system. However, the system satisfies homogeneity: if we replace the input $x(n)$ with $c x(n)$ then the output is $c y(n)$. To check time invariance, apply the input $x_{1}(n)=x(n-N)$. Then the output is

$$
y_{1}(n)= \begin{cases}x_{1}^{2}(n) / x_{1}(n-1), & \text { if } x_{1}(n-1) \neq 0 \\ 0, & \text { if } x_{1}(n-1)=0 .\end{cases}
$$

Substituting $x_{1}(n)=x(n-N)$, this yields

$y_{1}(n)= \begin{cases}x^{2}(n-N) / x(n-N-1), & \text { if } x(n-N-1) \neq 0 \\ 0, & \text { if } x(n-N-1)=0\end{cases}$

which is equal to $y(n-N)$, proving time invariance. Thus the system (2) is HTI but not LTI. Note that the exponential input $x(n)=a^{n}$ produces $y(n)=a^{2 n} / a^{n-1}=a \times a^{n}$, which shows that $H(a)=a$.

Continuous-Time HTI Systems: A similar argument holds for continuous-time HTI systems as well, with a minor adjustment of details. Assume that an input of the form $x(t)=e^{a t}$ produces the output $y(t)$. By time-invariance the shifted input $x_{1}(t)=e^{a(t-\tau)}$ produces the output $y(t-\tau)$. But the shifted input can also be written as $x_{1}(t)=e^{-a \tau} e^{a t}$ which shows, by homogeneity, that the output is $e^{-a \tau} y(t)$. The uniqueness 
of the output therefore implies $y(t-\tau)=e^{-a \tau} y(t)$ for all $t$. Setting $t=\tau$ we obtain $y(0)=e^{-a \tau} y(\tau)$, or equivalently $y(\tau)=e^{a \tau} y(0)$ for any choice of $\tau$. This proves that the output is indeed of the form $y(t)=y(0) e^{a t}$.

\section{IMPULSE RESPONSE AND FREQUENCY RESPONSE}

It is probably clear to the reader that the concepts of impulse response and frequency response are not useful for HTI systems. To drive home the point, consider the HTI system (2) again, and apply the input $x(n)=\delta(n)$. Since $\delta(n)$ and $\delta(n-1)$ cannot both be nonzero for any $n$, it follows that the impulse response $h(n)=0$ for all $n$. This is another proof that the system is not LTI, for if it were, then the output would be zero for all inputs. Evidently the concept of an impulse response is not very useful for HTI systems.

Since an HTI system produces the output $y(n)=H(a) a^{n}$ in response to the input $x(n)=a^{n}$, we see that the input $x(n)=e^{j \omega_{0} n}$ produces the output $H\left(e^{j \omega_{0}}\right) e^{j \omega_{0} n}$. Perhaps it is tempting to interpret $H\left(e^{j \omega}\right)$ as the frequency response. For the example shown in (2) the frequency response would then be

$$
H\left(e^{j \omega}\right)=e^{j \omega} .
$$

Since $\left|H\left(e^{j \omega}\right)\right|=1$ this behavior resembles that of an allpass system. [In fact, an LTI system with this frequency response would have been an advance operator $H(z)=z$.] Summarizing, the frequency response of the HTI example (2) resembles that of an allpass filter, whereas the impulse response $h(n)$ is zero for all $n$. This demonstrates that $h(n)$ and $H\left(e^{j \omega}\right)$ do not form a Fourier transform pair for HTI systems. For the example (2), the single frequency input $x_{0}(n)=1=e^{j 0 n}$ produces the output $y_{0}(n)=1$ for all $n$. If we apply the input $x_{\pi}(n)=(-1)^{n}=e^{j \pi n}$ the output would be $y_{\pi}(n)=-e^{j \pi n}$. If we now apply the superposition

$$
x_{0}(n)+x_{\pi}(n)= \begin{cases}2, & \text { for even } n \\ 0, & \text { for odd } n\end{cases}
$$

then the output of (2) is $y(n)=0$ for all $n$, which is not the sum of $y_{0}(n)$ and $y_{\pi}(n)$. In fact, the responses at the two frequencies appear to have cancelled each other off! Evidently, the frequency response concept is not useful because there is no superposition property.

\section{CONCLUding REMARKS}

It will be nice to find out if there is an even larger class of systems (i.e., more general than HTI) with the exponential eigenfunction property. In particular, can we find a simple description for the entire class of systems with this property? This appears to be an important question of academic interest.

\section{ACKNOWLEDGMENT}

The observations presented here came out of the author's teaching experience at California Institute of Technology, especially basic courses in signals and systems. In this regard, very insightful discussions with Prof. J. Franklin are gratefully acknowledged, as are the discussions with graduate students M. Mandel and M. Mese.

\section{REFERENCES}

[1] A. V. Oppenheim and A. S. Willsky (with S. Hamid Nawab), Signals and Systems. Englewood Cliffs, NJ: Prentice-Hall, 1997.

[2] L. B. Jackson, Signals, Systems, and Transforms. Reading, MA: Addison-Wesley, 1991. 